\title{
Burnout en médicos residentes de especialidades y subespecialidades: estudio de prevalencia y variables asociadas en un centro universitario
}

\author{
Luis Antonio Díaz ${ }^{1}$, Juan Pablo Arab ', Carolina Núñez ', Camila Robles ', Marcela Bitran ${ }^{\text {, }}$, María Pía Nitsche 1, Daniela Véliz 1 ,

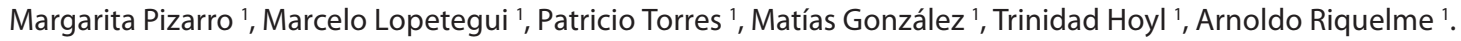

Resumen: Introducción: El burnout es un síndrome caracterizado por agotamiento emocional, despersonalización y bajo sentido de logro personal. Los médicos residentes de especialidad y subespecialidad constituyen una población de riesgo por la alta carga laboral y la interferencia con su vida personal. Nuestro objetivo fue evaluar la prevalencia de burnout y su asociación con variables sociodemográficas, en residentes de especialidad y subespecialidad de la Pontificia Universidad Católica de Chile (PUC).

Métodos: Se realizó una encuesta electrónica a los residentes de especialidad y subespecialidad de la PUC, que incluyó el “Inventario de Burnout de Maslach" (22 preguntas divididas en 3 dimensiones). Se sumaron los puntos de cada dimensión y se clasificó a los residentes en riesgo de burnout al presentar altos índices de agotamiento emocional y/o despersonalización. El análisis estadístico incluyó un análisis univariado y multivariado.

Resultados: 415 encuestas fueron contestadas (tasa de respuesta 86\%). El 38,3\% de los residentes cumplió criterios de burnout, con un $41,9 \%$ en residentes de especialidad y $24,1 \%$ en residentes de subespecialidad. En el análisis por subgrupos, la mayor prevalencia se encontró en especialidades quirúrgicas (55,3\%). Los residentes extranjeros, los programas de especialidad (comparados con subespecialidad) y los programas de especialidades quirúrgicas se asociaron de manera independiente a burnout (OR 3,8 IC95\% 1,4-10,5, $\mathrm{p}=0,01 ;$ OR 2,3 IC95\% 1,3-4,1, p<0,01 y OR 1,7 IC95\% 1,1-2,7; $\mathrm{p}=0,02$, respectivamente). La carga laboral horaria no se asoció de manera independiente a burnout $(\mathrm{p}=0,19)$.

Conclusión: Los residentes de especialidad y subespecialidad presentan una alta prevalencia de burnout. Adicionalmente, ser extranjero, el pertenecer a un programa de especialidad y los programas de especialidades quirúrgicas se asocian de manera independiente a burnout.

Palabras clave: burnout; residentes; médicos; especialidad; subespecialidad; sobrecarga horaria.

Abstract: Introduction: Burnout is a pathological syndrome characterized by emotional exhaustion, depersonalization and low sense of personal accomplishment. Residents from medical specialties and subspecialties constitute a population at risk for high work overload and interference in personal life. The aim of this study was to evaluate the prevalence of burnout and its associations with sociodemographic variables, in specialty and subspecialty residents of the Pontificia Universidad Católica de Chile (PUC).

Methods: An electronic survey was answered by residents of specialty and subspecialty of PUC. It included the "Maslach Burnout Inventory" (which consists of 22 questions divided into 3 dimensions). The points of each dimension were added and burnout was defined as a high score on depersonalization or emotional exhaustion subscales. Statistical analysis included an univariate and multivariate analysis. Results: 415 surveys were answered (response rate $86 \%$ ). 38.3\% of residents met criteria for burnout, with a percentage of $41.9 \%$ for specialty residents and $24.1 \%$ for subspecialty residents. In the subgroup analysis, the highest prevalence was found in surgical specialties (55.3\%). Foreign residents, medical residency programs (compared to sub specialization programs) and surgical programs were independently associated with burnout (OR 3.8 IC95\% 1.4-10.5, p=0.01; OR 2.3 IC95\% 1.3-4.1, p<0.01 y OR 1.7 IC95\% 1.1-2.7; p=0.02, respectively). There was no independent association between duty hours and burnout $(p=0.19)$.

Conclusion: Specialty and subspecialty residents have a high prevalence of burnout. Additionally, foreign residents, participation in a speciality residency (compared to subspecialties programs) and surgical residencies are independently associated to burnout.

Keywords: Burnout; residents; physicians; postgraduate; duty hours.

Fecha de envío: 18 de enero de 2017 - Fecha de aceptación: 16 de agosto de 2017

(1) Escuela de Medicina, Pontificia Universidad Católica de Chile. Santiago de Chile.

*Autores de correspondencia:a.riquelme.perez@gmail.com /mthoyl@med.puc.cl 


\section{Introducción}

La calidad de vida y bienestar de los médicos es un tema ampliamente discutido. Específicamente, el período de especialización y subespecialización posee un elevado número de factores de riesgo y de desafío profesional, puesto que implica responder a la carga asistencial, un vasto número de responsabilidades, actualización de conocimientos teóricos, jornadas extensas con exceso de horas de trabajo semanal, baja sensación de control de las situaciones, y al mismo tiempo, la necesidad de construir un proyecto de vida personal y la interferencia del mundo profesional en el hogar (Thomas 2004).

A partir de la observación, descripción y análisis psicológico de un patrón de conducta en trabajadores que se desempeñaban en servicios de atención al público, surgió el concepto de burnout. Este fenómeno, descrito de manera independiente por Freudenberger y Maslach, es una metáfora para expresar agotamiento del trabajador, entendido como el sofocamiento de un incendio o una vela, donde ya no existen suficientes recursos para mantener el fuego encendido (Freudenberger 1974; Maslach 1976, Schaufeli et al. 2009). El burnout constituye un síndrome caracterizado por 3 dimensiones: agotamiento emocional (manifestado como bajo entusiasmo por el trabajo, sensación de impotencia y de estar atrapado y/o derrotado); despersonalización (caracterizado por un trato indiferente a pacientes y una actitud negativa frente a colegas y a la profesión); y baja sensación de realización personal (desapego por el trabajo y evasión de las responsabilidades) (Maslach et al. 1996). Estas 3 dimensiones pueden encontrarse en distintos grados, dándole un carácter heterogéneo y continuo al síndrome. Si bien el síndrome de burnout tiene un origen multifactorial, el estrés ocupacional prolongado se ha identificado ampliamente como su principal causa. Además, altas demandas en el trabajo podrían jugar un rol particular en el desarrollo del síndrome (Seidler et al. 2014).

El burnout es frecuente en el ámbito médico. Esto ha sido documentado por múltiples estudios que muestran que un tercio de los médicos han presentado burnout en algún punto de su carrera. Un estudio de burnout en EE. UU. Mostró una prevalencia de 54,4\% entre los médicos residentes (Shanafelt et al. 2015).

En dicho estudio, se describe una prevalencia de $50 \%$ y $76 \%$ para residentes de cirugía y medicina interna, respectivamente. Además, los residentes tienen significativamente más riesgo de burnout que la población general, aún luego de ajustarlo por sexo, edad, horas de trabajo y nivel educacional (OR 1,97, IC 95\% 1,80-2,16, p<0,001). Adicionalmente, la prevalencia en este grupo ha aumentado hasta en un 10\% en los últimos 3 años en EE. UU. (Shanafelt et al. 2015).

El estudio de burnout en residentes ha sido de gran interés debido a varios aspectos. Uno de ellos es el impacto que produce en la calidad y resultados de la atención de salud. Esto se debe a que el burnout altera la salud mental y disminuye la calidad de vida de residentes, lo que se traduce en menos horas de trabajo productivas y aumento en el riesgo de errores médicos, que generan costos y consecuencias asociadas (Zis et al. 2015). Asimismo, el burnout también afecta la función cardíaca y su neurroregulación, pudiendo constituir un factor independiente de riesgo cardiovascular (Cursoux et al. 2012).

En la literatura existen numerosos estudios en relación al burnout en residentes, que buscan conocer la prevalencia de este, sus factores predisponentes y de riesgo, las consecuencias a nivel de salud y de qué manera se puede contribuir a una mejora de la situación. No obstante, no contamos con datos actuales de prevalencia en nuestro país. Por lo tanto, el objetivo de nuestro trabajo fue evaluar la prevalencia de burnout en residentes de especialidades y subespecialidades en la Pontificia Universidad Católica de Chile.

\section{Materiales y métodos}

Se aplicó una encuesta que evaluó las características sociodemográficas y aspectos académicos de los residentes de especialidades y subespecialidades de la Pontificia Universidad Católica de Chile.

El instrumento se aplicó vía correo electrónico a todos los residentes de especialidad y subespecialidad, entre los meses de julio y septiembre del año 2013. Este período está libre de exámenes para todos los residentes y corresponde a 3 a 5 meses desde el inicio de la especialización para los residentes de primer año. Se contó con un plazo de 2 meses para contestarla. Se obtuvo consentimiento informado de todos los participantes.

Para el estudio se consideraron los residentes de especialidades y subespecialidades en grupos diferentes, categorizando en áreas médica, quirúrgica, médico-quirúrgica o de gestión y diagnóstico en las especialidades, y médica o quirúrgica en las subespecialidades de la forma en que se presenta en las Tablas 1 y 2. 
Tabla 1: Especialidades incluidas en el estudio y su división en grupos según tipo de actividad en médicas, quirúrgicas, médico-quirúrgicas, diagnóstico y gestión.

\begin{tabular}{|c|c|c|c|c|}
\hline & $\begin{array}{c}\text { Médicas } \\
(n=10)\end{array}$ & Quirúrgicas ( $n=6$ ) & Médico-Quirúrgicas ( $\mathrm{n}=3$ ) & Diagnóstico y Gestión ( $n=5$ ) \\
\hline Especialidad & $\begin{array}{l}\text { - Medicina Familiar } \\
\text { (mención adulto) } \\
\text { - Medicina Familiar } \\
\text { (mención niño) } \\
\text { - Medicina Interna } \\
\text { - Medicina Urgencia } \\
\text { - Neurología } \\
\text { - Neurología Pediátrica } \\
\text { - Nutrición y Diabetes } \\
\text { - Pediatría } \\
\text { - Psiquiatría } \\
\text { - Radio-oncología }\end{array}$ & $\begin{array}{l}\text { - Anestesiología } \\
\text { - Cirugía general } \\
\text { - Neurocirugía } \\
\text { - Obstetricia y } \\
\text { Ginecología } \\
\text { - Traumatología y } \\
\text { Ortopedia } \\
\text { - Urología }\end{array}$ & $\begin{array}{l}\text { - Dermatología } \\
\text { - Oftalmología } \\
\text { - Otorrinolaringología }\end{array}$ & $\begin{array}{l}\text { - Anatomía Patológica } \\
\text { - Laboratorio Clínico } \\
\text { - Medicina Nuclear } \\
\text { - Radiología } \\
\text { - Salud Pública }\end{array}$ \\
\hline
\end{tabular}

Tabla 2: Subespecialidades incluidas en el estudio y su división en grupos según tipo de actividad en médicas y quirúrgicas.

\begin{tabular}{|l|l|l|}
\hline \multicolumn{1}{|c|}{ Médicas (n=17) } & \multicolumn{1}{|c|}{ Quirúrgicas (n=9) } \\
\hline & - Cardiología & \\
& - Cardiología Pediátrica & \\
& - Endocrinología del Adulto & \\
& - Endocrinología Pediátrica & - Anestesia Cardiovascular \\
& - Enfermedades Respiratorias Pediátrica & - Anestesia Regional y Analgesia Perioperatoria \\
& - Enfermedades Respiratorias del Adulto & - Cirugía cardiovascular \\
& - Gastroenterología del Adulto & - Cirugía digestiva \\
& - Gastroenterología y Nutrición Pediátrica & - Cirugía Oncológica de Cabeza y Cuello \\
& - Geriatría & - Cirugía Plástica y Reconstructiva \\
& - Hematología del Adulto & - Cirugía Vascular Periférica \\
& - Infectología Pediátrica & - Ginecología oncológica \\
& - Medicina Intensiva Adultos & - Ginecología Materno-infantil \\
& - Inmunología Clínica y Reumatología & \\
& - Medicina Intensiva Infantil & \\
& - Nefrología & \\
& - Neonatología & \\
& - Oncología Médica & \\
\hline
\end{tabular}

La encuesta incluyó características sociodemográficas, cantidad de horas de trabajo semanales presenciales (incluyendo turnos presenciales) y el cuestionario del inventario de Maslach (Maslach et al. 1996). Se eligió este instrumento dado que es el más utilizado en la literatura internacional y cuenta con una validación en residentes de especialidades y subespecialidades de habla hispana (cronbach $a=0,885$; instrumento multidimensional) (Rivera-Ávila et al. 2017). Este consta de 3 dimensiones medidas en un total de 22 ítems. La primera dimensión está constituida por 9 ítems y tiene como finalidad medir el agotamiento emocional, es decir, la sensación de estar sobreexigido o emocionalmente exhausto por el trabajo; la segunda consta de 5 ítems mediante los cuales se mide la despersonalización y el cinismo del individuo, poniendo énfasis en el grado de respuesta impersonal hacia los usuarios. Por último, la tercera dimensión cuenta con 8 ítems sobre la realización personal, evaluando sentimientos de competencia y logros del trabajo del sujeto. Se consideró sugerente de burnout un alto agotamiento emocional ( $\geq 27$ puntos) y/o despersonalización ( $\geq 10$ puntos) (Krasner et al. 2009). La realización personal fue analizada por separado, ya que algunos estudios sugieren que esta constituiría una dimensión parcialmente distinta al burnout. Se consideró una baja realización personal con puntaje $\leq 33$ puntos (Shanafelt et al. 2002). 
Diaz et al.

El análisis estadístico incluyó Chi-cuadrado, $t$-Student y un análisis multivariado mediante regresión logística. Se utilizó el software SPSS v22,0 (Armonk, NY: IBM Corp.). Los datos se utilizaron de manera anónima y los analistas fueron externos a la Dirección de Postgrado y los programas de formación de nuestra institución. Además, se obtuvo la aprobación del Comité de Ética de la Pontificia Universidad Católica de Chile, quienes otorgaron una dispensa de consentimiento.

\section{Resultados}

Se obtuvo respuesta de 415 participantes (con una tasa de respuestas de $86 \%$ del universo considerado), de las cuales $50,8 \%$ corresponden a sexo femenino y $49,2 \%$ a sexo masculino. De estas, 332 encuestas corresponden a residentes realizando especialidad (80\%) y 83 a residentes realizando subespecialidad (20\%). La edad promedio de respuesta fue 29,5 años, con un rango de 25 a 50 años.

Tabla 3: Variables demográficas consideradas en el estudio (edad, sexo, estado civil, nacionalidad, universidad, tipo de residencia y año de residencia) y sus porcentajes respectivos.

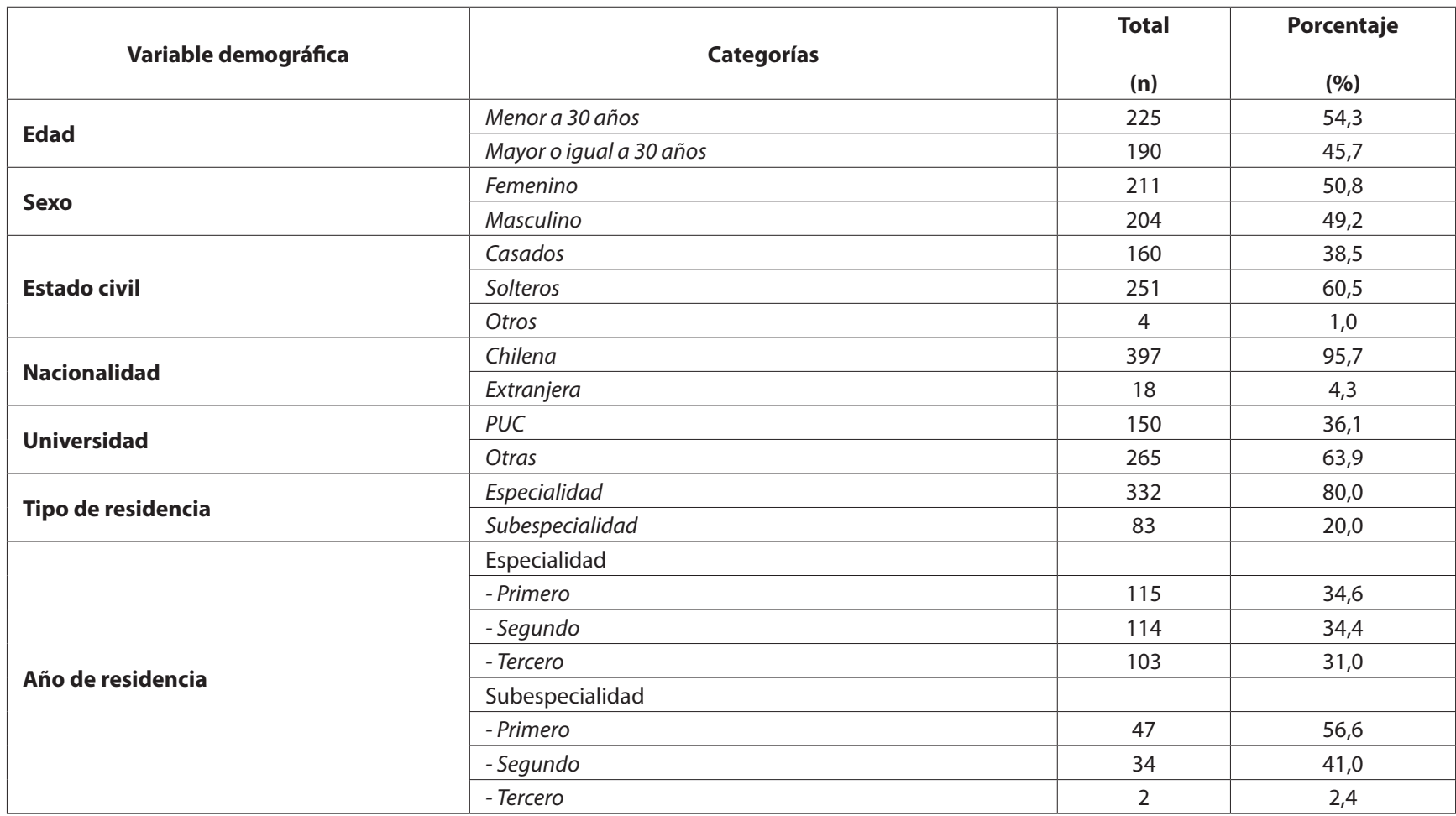

El 4,3\% de los residentes eran extranjeros. En la Tabla 3 se detallan las variables demográficas estudiadas y sus valores respectivos.

Al evaluar la presencia de burnout en residentes, se obtuvo una prevalencia general de 38,3\%. La prevalencia fue significativamente mayor en residentes extranjeros que en chilenos $(66,7 \%$ versus $37,0 \%$, respectivamente; $\mathrm{p}=0,11)$. Además, se observó una baja realización personal en el $24,3 \%$ de los residentes (Figura 1). En el análisis según sexo, se obtuvo una prevalencia de $42,2 \%$ para sexo femenino y $34,3 \%$ para sexo masculino, sin diferencias estadísticamente significativas entre ellas $(p=0,099)$. Asimismo, al evaluar la presencia de burnout en residentes según edad y según estado civil (casados versus no casados), no hubo diferencias significativas ( $p=0,878$ y $p=0,316$, respectivamente).

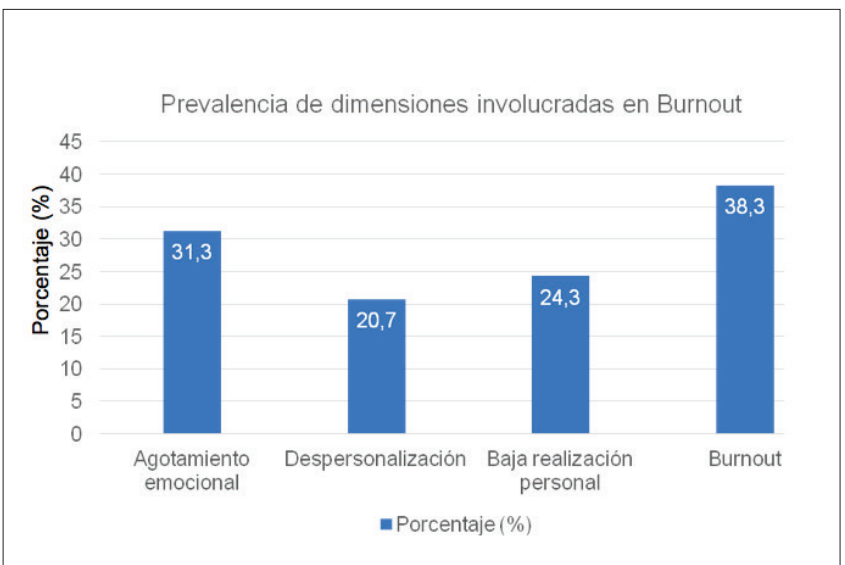

Figura 1: Prevalencia de agotamiento emocional, despersonalización, baja realización personal y burnout en residentes de especialidad y subespecialidad. 
Al realizar el análisis por tipo de residencia, se obtuvo una prevalencia de burnout de 41,9\% para residentes realizando especialidad y de $24,1 \%$ para subespecialidad, cuya diferencia es estadísticamente significativa $(p=0,003)$.

En la evaluación según grupos de especialidades, se obtuvo que la prevalencia de burnout de especialidades quirúrgicas es 55,3\%; la de especialidades médicas es 39,2\%; las de diagnóstico y de laboratorio es $35,7 \%$ y finalmente las especialidades médico-quirúrgicas presentan una prevalencia de $23,3 \%(p=0,058)$ (Figura 2). Asimismo, en el análisis por subespecialidad, se obtuvo que la prevalencia de burnout entre los residentes de subespecialidades quirúrgicas en comparación con los residentes de subespecialidades médicas de $13,6 \%$ y $27,9 \%$, respectivamente, sin diferencias estadísticamente significativas $(p=0,181)$.

Al evaluar la prevalencia de burnout por año de residencia, se obtuvo que en primer año esta correspondía a 34,6\%, en segundo año a $37,8 \%$ y en tercer año a $44,8 \%$, sin diferencias estadísticamente significativas entre ellas $(p=0,244)$.

Al realizar el análisis según especialidad/subespecialidad y carga horaria, se observó que las especialidades quirúrgicas tenían significativamente mayor porcentaje de burnout $(55,3 \%)$ y al mismo tiempo, mayor número de horas de trabajo semanal en promedio (89,6 horas). Por su parte, las especialidades médico-quirúrgicas que presentaban el menor porcentaje de burnout (23,3\%) tenían el menor número de horas de trabajo semanal (60,1 horas) (Figura 2). Adicionalmente, la carga laboral horaria (incluyendo turnos presenciales) se asoció a burnout en el análisis univariado $(p=0,23)$.

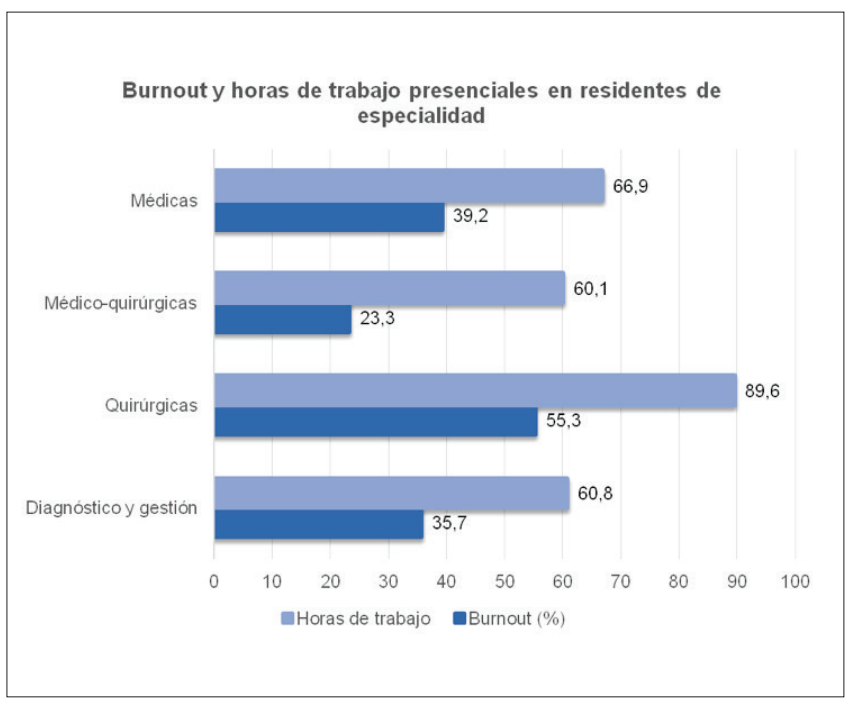

Figura 2: Relación entre Burnout y carga horaria semanal en programas de especialidad. Las horas de trabajo semanal incluyen los turnos presenciales.
Basados en estos hallazgos, se realizó un análisis multivariado que incluyó nacionalidad (chilena versus extranjero), carga laboral horaria, tipo de programa (especialidad versus subespecialidad) y tipo de especialidad (quirúrgica versus no quirúrgica). Como resultado, se observó que ser extranjero se asoció de manera independiente a burnout (OR 3,8 IC95\% 1,4-10,5; p=0,01). Además, el pertenecer a un programa de especialidad (comparado con subespecialidad) se asoció de manera independiente a burnout (OR 2,3 IC95\% 1,3-4,1; $\mathrm{p}=0,003)$. Los programas de especialización quirúrgicos también se asociaron de manera independiente a burnout (OR 1,7 IC95\% $1,1-2,7 ; p=0,02)$. La carga laboral horaria no se asoció de manera independiente a burnout $(p=0,19)$.

\section{Discusión}

En este estudio se demostró que el burnout es un problema frecuente entre los residentes tanto de especialidades como de subespecialidades, siendo más frecuente en las primeras. El impacto negativo que tiene el burnout en profesionales de la salud es de vital importancia, pues se ha demostrado que existe una relación significativa entre los profesionales con burnout y la tasa de errores que se cometen, comprometiendo así la seguridad y calidad de atención del paciente (Prins et al. 2009).

La proporción de residentes con burnout en nuestro estudio es menor a las publicadas en otros estudios de similares características, donde se encontró que un 50\% de los residentes de especialidades variadas presentaban algún criterio de burnout (Martini et al. 2004). Sin embargo, hay que considerar que estos estudios han sido realizados en otros países con sistemas educacionales diferentes al de Chile. Hasta la fecha, no existe información local sobre el burnout en residentes de especialidades específicas en Chile; en este trabajo presentamos los primeros datos chilenos de prevalencia de burnout en residentes de variadas especialidades y subespecialidades.

En la literatura se describe que la prevalencia del burnout en los programas de residencia tiende a ser mayor en los residentes de primer año, para luego disminuir y mantenerse durante los siguientes años de estudio; se plantea que esto podría ocurrir debido a un proceso de adaptación espontáneo durante los años de formación a las nuevas responsabilidades, presiones emocionales y distintos aspectos de la vida laboral (Martini et al. 2004). Sin embargo, en nuestro estudio no se observaron diferencias estadísticamente significativas entre la prevalencia de burnout según los distintos años de residencia. Los datos son insuficientes para otorgar una explicación a estas incongruencias. 
Respecto a los residentes extranjeros, estos presentaron una prevalencia significativamente mayor de burnout. Aunque existen instancias de inducción y capacitación para los residentes extranjeros en nuestra institución, esta prevalencia también se podría explicar por el desafío que supone adaptarse a un sistema de salud distinto, junto con la idiosincrasia de otro país.

Por otro lado, las especialidades quirúrgicas se asociaron de manera independiente a mayor burnout entre sus residentes, en comparación con las médico-quirúrgicas, médicas o de laboratorio. Esto pudiese estar determinado por características curriculares específicas de estos programas, no obstante, se necesitan estudios adicionales para indagar respecto a la mayor prevalencia observada en este grupo.

Estudios previos han demostrado que la carga de trabajo y una elevada cantidad de horas laborales son factores de riesgo para desarrollar burnout (Martini et al. 2004). En nuestro estudio se obtuvo una relación proporcional entre la cantidad de horas de trabajo presenciales con el grado de burnout de los residentes. Sin embargo, esta relación no se asoció de manera independiente a burnout en el análisis multivariado.

Se sabe que el burnout es una condición multifactorial, en la cual afectan tanto rasgos de personalidad individuales y habilidades para enfrentar el estrés, como las características generales de la situación laboral a las que la persona se ve expuesta, entre las cuales se encuentran la sobrecarga laboral, bajo salario, falta de supervisión, ambigüedad de roles y variable propias de la institución laboral (Lue et al. 2010).

En cuanto a las intervenciones, parece más importante y más efectivo preferir medidas preventivas a las paliativas para disminuir las tasas de burnout. Tomando en cuenta que los residentes del primer año son los más afectados por esta patología, una intervención adecuada podría ser encontrar una manera de preparar a los alumnos de pregrado en habilidades de coping y manejo del estrés, de tal forma de entregarles las herramientas necesarias para enfrentarse al mundo laboral. Otra medida útil sería disminuir el número de horas de trabajo, evaluando formas de hacerlas más efectivas y menos estresantes.

Se sabe que la salud mental positiva influye de manera favorable en mejorar conductas profesionales y en desarrollar el altruismo con los pacientes y la sociedad. Además, se ha visto que tiene un efecto preventivo en el burnout de estudiantes de medicina. En un trabajo sobre este tema, se comenta la importancia que tiene en la educación médica no solo prevenir el burnout, sino también promover el autoconocimiento, el pertenecer a grupos, el crear lazos de amistad y autocuidado, como herramientas para lograr una salud mental positiva (Pedrals et al. 2011; Dyrbye et al. 2012). Dentro de los factores que influyen en esta, se encuentra el bienestar social, que tiene que ver con cómo la persona se desenvuelve en la sociedad, medible con el cuestionario de Keyes (Dyrbye et al. 2012). Aplicar este instrumento a nuestros residentes podría complementar los resultados de este trabajo, aportando una idea sobre cómo intervenir para mejorar la calidad de vida de los mismos. Adicionalmente, existe diversa evidencia experimental que apoya al deporte, la gratitud y el cultivo de mindfulness o "atención plena" como actividades potenciadoras del bienestar subjetivo de forma sostenida, las que podrían ser útiles en nuestros programas de formación (Shapiro et al. 1998; Seligman et al. 2005; Finkelstein et al. 2007).

Otro aspecto fundamental es el ambiente educacional, el cual ha sido evaluado en nuestra institución (Herrera et al. 2012). Dentro de los aspectos que los residentes manifiestan que se pueden mejorar, se mencionan la falta de acceso a consejería durante el programa de residencia y en particular, en momentos de dificultad académica. Estos hallazgos podrían estar jugando un rol en la prevalencia de burnout; por lo tanto, el fomentar medidas de apoyo académico e instancias de consejería y acompañamiento del residente, podrían ser medidas útiles para disminuir la prevalencia a mediano y largo plazo.

La baja realización personal fue un aspecto llamativo en nuestros resultados, el cual estuvo presente en 1 de cada 4 residentes. Consideramos que es importante realizar estudios de investigación con metodología cualitativa para indagar respecto a las causas de dicho fenómeno. Asimismo, se deben buscar de manera exhaustiva aquellos factores asociados al estrés ocupacional, a la baja realización personal y al síndrome de burnout. Este punto constituye un gran desafío a futuro, el cual nos permitirá implementar medidas específicas con el fin de mejorar el bienestar de nuestros residentes.

\section{Conclusiones}

El burnout es un síndrome prevalente, y los residentes tanto de especialidad como de subespecialidad se encuentran en un riesgo elevado de padecerlo. La importancia de estudiarlo se basa en los costos y consecuencias que trae, tanto para la persona que lo padece como para el sistema de salud. Existe en general, una mayor prevalencia de burnout en especialidad, especialmente en el ámbito quirúrgico, y en residentes extranjeros. Es esencial identificar predisponentes y factores asociados, para implementar medidas de prevención de burnout y asimismo, pesquisar a aquellos residentes con burnout para realizar una intervención oportuna. 


\section{Referencias}

Cursoux P, Lehucher-Michel MP, Marchetti H, Chaumet G \& Delliaux $S$ (2012). Burnout syndrome: a true cardiovascular risk factor. Presse Med 41, 1056-1063.

Dyrbye L, Harper W, Moutier C, Durning S, Power D, Massie S, Eacker A, Thomas M, Satele D, Sloan J \& Shanafelt T (2012). A Multi-institutional Study Exploring the Impact of Positive Mental Health on Medical Students. Professionalism in an Era of High Burnout. Academic Medicine 87, 1024-1031.

Finkelstein C, Brownstein A, Scott C \& Lan Y (2007). Anxiety and stress reduction in medical education: an intervention. Medical Education 41, 258-264.

Freudenberger H (1974). Staff burnout. Journal of Social Issues 30 , 159-165.

Herrera C, Olivos T, Román J, Larraín A, Pizarro M, Solís N, Sarfatis A, Torres P, Padilla O, Le Roy C \& Riquelme A. (2012). Evaluación del ambiente educacional en programas de especialización médica. Revista médica de Chile 140, 1554-1561.

Krasner MS, Epstein RM, Beckman H, Suchman AL, Chapman B, Mooney CJ \& Quill TE (2009). Association of an educational program in mindful communication with burnout, empathy, and attitudes among primary care physicians. JAMA 302, 1284-1293.

Lue B, Chen H, Wang C, Cheng Y \& Chen M (2010). Stress, personal characteristics and burnout among first postgraduate year residents: a nationwide study in Taiwan. Med Teach 32, 400-407.

Martini S, Arfken C, Churchill A \& Balon R (2004). Burnout comparison among residents in different medical specialties. Academic psychiatry 28, 240-242.

Maslach, C. (1976). Burned-out. Human Behavior 9, 16-22.

Maslach C, Jackson SE \& Leiter MP (1996). Maslach Burnout Inventory. Third edition. Palo alto, California. Consulting Psychologist Press.

Pedrals N, Rigotti A, \& Bitran M. (2011). Aplicando psicología positiva en educación médica. Revista médica de Chile 139, 941-949.
Prins J, van der Heijden F, Hoekstra-Weebers J, Bakker A, van de Wiel $H$, Jacobs B \& Gazendam-Donofrio S (2009). Burnout, engagement and resident physicians' self-reported errors. Psychology, health \& medicine 14, 654-666.

Rivera-Ávila DA, Rivera-Hermosillo JC \& González-Galindo C (2017). Validación de los cuestionarios CVP-35 y MBI-HSS para calidad de vida profesional y burnout en residentes. Investigación en Educación Médica 6, 25-34.

Schaufeli W, Leiter M \& Maslach C (2009). Burnout: 35 years of research and practice. Career Development International 14, 204-220.

Seidler A, Thinschmidt M, Deckert S, Then F, Hegewald J, Nieuwenhuijsen K \& Riedel-Heller S (2014). The role of psychosocial working conditions on burnout and its core component emotional exhaustion - a systematic review. Journal of occupational medicine and toxicology $9,10$.

Seligman MEP, Steen TA, Park N \& Peterson C (2005). Positive psychology progress. Empirical validation of interventions. Am Psychol 60, 410-421.

Shanafelt TD, Bradley KA, Wipf JE \& Back AL (2002). Burnout and self-reported patient care in an internal medicine residency program. Ann Intern Med. 136, 358-367.

Shanafelt T, Hasan O, Dyrbye L, Sinsky C, Satele D, Sloan J \& West C (2015). Changes in burnout and satisfaction with work-life balance in physicians and the general US working population between 2011 to 2014. Mayo Clin Proc 90, 1600-1613.

Shapiro SL, Schwartz GE \& Bonner G (1998). Effects of mindfulness-based stress reduction on medical and premedical students. J Behav Med 21, 581-599.

Thomas N (2004). Resident burnout. JAMA 292, 2880-2889.

Zis P, Artemiadis A, Lykouri M, Xiron S, Roussopoulou A,Papaglorgion $E$, Bakola $E$ \& Anagnostopoulos $F$ (2015). Residency training: Determinants of Burnout of neurology trainees in Attica, Greece. Neurology 85, 81-84. 